\title{
Advantages of lean techniques application in apparel industry: case study on knit jacket
}

\begin{abstract}
It is proven that by the application of Lean techniques in manufacturing, business can be profited by improvement in the level of productivity and cutting down the processes that is responsible for wastages. In Bangladesh, Apparel industries face a lot of challenges and the most difficult of them is to meet the shipment date. To ensure the products have been manufactured and assembled in due time, manufacturers emphasize on choosing the best method of production process. With the help of Kaizen and 5's, it is possible to identify non value added processes and eliminate them from the production process. In this paper, we have taken the production data of a knitted jacket and considered the SMV data in two phases, one with the traditional line and the other one is with the implementation of Lean technique to see the differences of SMV data in different stages of production.
\end{abstract}

Keywords: SMV, lean, kaizen, 5'S, line target, line efficiency
Volume 5 Issue 5 - 2019

\author{
Shah MD. Maruf Hasan,' MD. Mustafizur \\ Rahman Shanta, ${ }^{2}$ Abdullah Al Shams, ${ }^{3}$ Md. \\ Sakibur Rahman, ${ }^{4}$ Sazid Elahi,' MD. Mazedul \\ Islam ${ }^{5}$ \\ 'Lecturer, Department of Apparel Engineering, Bangladesh \\ University of Textiles, Bangladesh \\ ${ }^{2}$ Executive, Industrial Engineering Department, Fakir Apparels \\ Ltd, Bangladesh \\ ${ }^{3}$ Trainee merchandiser, $\mathrm{Ha}$-Meem group, Bangladesh \\ ${ }^{4}$ Executive, Research \&Development, Square Fashions Ltd, \\ Bangladesh \\ ${ }^{5}$ Assistant professor, Department of Apparel Engineering, \\ Bangladesh University of Textiles, Bangladesh
}

Correspondence: MD. Maruf Hasan, Lecturer, Department of Apparel Engineering, Bangladesh University of Textiles, Tejgaon, Dhaka-I208, Bangladesh, Email marufbutex39ae@gmail.com

Received: September 23, 2019 | Published: October |I, 2019

\section{Introduction}

Apparel industries from all over the world faced a great deal of negative impact due to the economic recession back in 2008 . And because of this the low cost garments had been urged by most consumer bases from all over the world. Then renowned apparel brands have been forced to cut down the prices to keep their products in the market. They have been shifted their vendors to low cost worker base countries like Bangladesh to keep the competition worldwide. To meet the global challenge, it is really vital to keep the production process in such a way that will not incorporate any types of waste and non-value added process when apparel production process is carried out with lean approach. The terminology is not that much unfamiliar to the manufacturers but they lack in consciousness about the strategic advantages that can be found while lean technique is used in apparel production which is the purpose of our study as well. ${ }^{1-8}$

\section{Objectives of the Study}

a. To find out the strategic advantages of lean technique in apparel industry.

b. To compare production data in terms of SMV target fulfilment, line efficiency, bottlenecks, capacity utilization in both casestraditional production line and lean production line.

c. To compare the productivity factors like transportation, inventory analysis, space utilization, defects analysis in both traditional line and lean line.

\section{Methodology}

For comparing productivity, we collected data from sewing floor of Adury Apparels Ltd, a sister concern of Thermax Group. We considered two lines (traditional \& lean line) \& differentiate between them. To calculate standard time for each operation, time study is conducted in the shop floor. To do this, a knit jacket is selected as a base line because operations differ from style to style and it is difficult to correlate all these operations of individual styles. After that, at least two operators were selected for each operation so that the difference in timing can be cross checked from the observed data of these two operators. To get better results, each operation time is taken for at least 5 cycles. Once time study is made by collecting raw data the performance rating is given to each operator and actual time is calculated for particular operation. Finally the Personal Fatigue and Delay (PFD) component as an allowance is added on the calculated time and the operation time is standardized. For calculation we have used the following formulas:

i. $\mathbf{S M V}=$ Basic time + Bundle Handling time + Allowance.

ii. Basic time $=$ Cycle time $\times$ Rating.

iii. Cycle time $=$ Pick up time + Stitching time + Dispose time.

iv. Efficiency\% of line $=($ Total production $\times \operatorname{smv} \times 100) /($ No of operator $\times$ working Hour $\times 60)$.

v. Basic pace time (B.P.T) $=$ Total time $/$ total manpower

\section{Research activities}

a) Become acquainted about Lean Technique

b) Vigorous study on Lean manufacturing tools

c) Select a factory for application

d) Observe Lean application on a particular floor 
e) Select a particular style to develop case study

f) Analysis Lean and Traditional line

g) Collect the necessary data (Figure 1) (Figure 2).

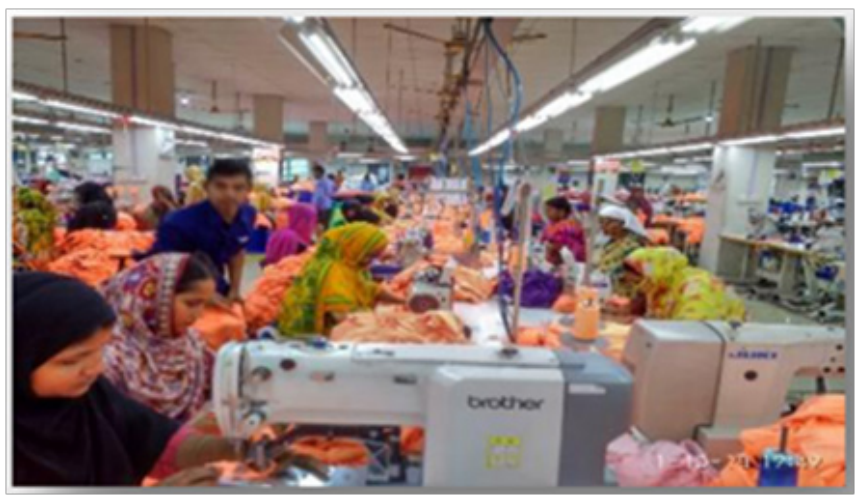

Figure I Lean line at adury apparel.

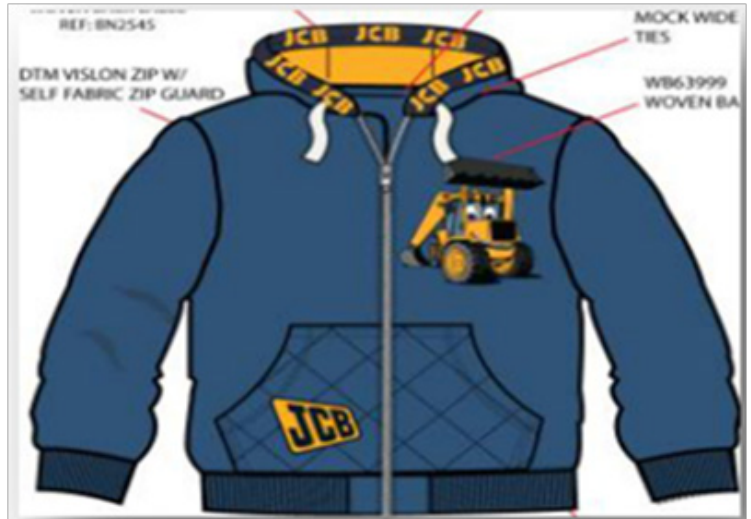

Figure 2 Knit jacket.

\section{Results and discussions}

We use time study to balance these sewing lines which is a part of work study. It implements the use of SMV calculation to identify the points where production has gone below the standard level and the places where the production is above the standard. Then it is balanced to remove bottle neck in order to increase productivity. This system was effective and helpful. Considerable improvement observed by using time study as a line balancing technique changing form traditional layout to balanced layout model. The exchanges of work between the operator \& helper caused a significant change in line results of reducing wastage of time, minimum no. of worker and which caused high productivity in the manufacturing process. This balancing process also leads to increased output per day, labor productivity, machine productivity and overall line efficiency.

\section{Lean line operation breakdown (Table I)}

Productivity: output $/$ input $\times 100 \%=78 / 100 \times 100 \%=78 \%$

SMV: $896.44 / 60=14.94$

Standard SMV: 12.77

SMV increased: $(14.94-12.77) / 14.94 \times 100=16.99 \%$

Efficiency\% of line: (Total production SMV x 100)/ (No of Operator $\mathrm{x}$ working hourx60) $=(78 \times 14.94 \times 100) /(35 \times 1 \times 60)=55.49 \%$

SMV target fulfillment: $(100-78) / 100 \times 100 \%=100 \%-22 \%=78 \%$

Basic pace time (B.P.T): Total time/total manpower $=896.44 / 35$ $=25.61 \mathrm{sec}$

Capacity/hr: $3600 /$ B.P.T $=3600 \mathrm{pcs} / 25.61=140$

\section{Traditional operation breakdown of knitted jacket} (Table 2)

Productivity: output/input $\times 100=64 / 100 \times 100=64 \%$

SMV: $1013.88 / 60=16.89$

Standard SMV: 15.43

SMV increased: $(16.89-15.43) / 15.43 \times 100=9.46 \%$

Efficiency\% of line: (Total productionxsmvx 100)/(No of operator $x$ working Hourx60 $)=(64 \times 16.89 \times 100) /(42 \times 1 \times 60)=42.89 \%$

SMV target fulfillment: $(100-64) / 100 \times 100 \%=100 \%-36 \%=64 \%$

Basic pace time $($ B.P.T) $=$ Total time $/$ total man power $=1013.88 / 42$ $=24.14 \mathrm{sec}$.

Capacity $/ \mathrm{hr}=3600 / 24.14=149 \mathrm{pcs}$.

Table I Lean line operation breakdown

\begin{tabular}{|c|c|c|c|c|c|c|c|c|c|}
\hline \multirow{2}{*}{ SL no. } & \multirow{2}{*}{ Operation } & \multirow{2}{*}{$\begin{array}{l}\text { No. of } \\
\text { workers }\end{array}$} & \multirow{2}{*}{ Machine } & \multicolumn{2}{|c|}{ Standard SMV } & \multirow{2}{*}{$\begin{array}{l}\text { Actual time } \\
\text { Sec(Avg.) }\end{array}$} & \multirow{2}{*}{$\begin{array}{l}\text { Allowance } \\
(12 \%)\end{array}$} & \multirow{2}{*}{$\begin{array}{l}\text { Standard } \\
\text { Time(Sec) }\end{array}$} & \multirow{2}{*}{ Capacity } \\
\hline & & & & Manual & $M / c$ & & & & \\
\hline I & $\begin{array}{l}\text { Pocket Bone Mark \& corner } \\
\text { cut }\end{array}$ & 1 & MNL & 0.25 & & 17 & 1.44 & 18.44 & 195 \\
\hline 2 & Bone Attach for Pocket & I & SNLS & & 0.5 & 32 & 3.84 & 35.84 & 100 \\
\hline 3 & $\begin{array}{l}\text { Body Mark for Pocket \& } \\
\text { attach pocket }\end{array}$ & 1 & SNLS & & 0.33 & 18 & 2.16 & 20.16 & 178 \\
\hline 4 & Pocket Cut & 1 & $\mathrm{MNL}$ & 0.58 & & 35 & 4.2 & 39.2 & 91 \\
\hline 5 & Pocket Top Stitch & I & SNLS & & 0.4 & 27 & 3.24 & 30.24 & 119 \\
\hline 6 & $\begin{array}{l}\text { Bone Inside Tack \& Pocket } \\
\text { Top Stitch Lower }\end{array}$ & 1 & SNLS & & 0.8 & 51 & 6.12 & 57.12 & 63 \\
\hline 7 & $\begin{array}{l}\text { Pocket Bag Close Both } \\
\text { Side(2) }\end{array}$ & I & OL & & 0.4 & 27 & 3.24 & 30.24 & 119 \\
\hline
\end{tabular}


Table Continues...

\begin{tabular}{|c|c|c|c|c|c|c|c|c|c|}
\hline \multirow{2}{*}{ SL no. } & \multirow{2}{*}{ Operation } & \multirow{2}{*}{$\begin{array}{l}\text { No. of } \\
\text { workers }\end{array}$} & \multirow{2}{*}{ Machine } & \multicolumn{2}{|c|}{ Standard SMV } & \multirow{2}{*}{$\begin{array}{l}\text { Actual time } \\
\text { Sec(Avg.) }\end{array}$} & \multirow{2}{*}{$\begin{array}{l}\text { Allowance } \\
(12 \%)\end{array}$} & \multirow{2}{*}{$\begin{array}{l}\text { Standard } \\
\text { Time(Sec) }\end{array}$} & \multirow{2}{*}{ Capacity } \\
\hline & & & & Manual & $M / c$ & & & & \\
\hline 8 & $\begin{array}{l}\text { Pocket Bag Mouth Close \& } \\
\text { Scissoring }\end{array}$ & 1 & SNLS & & 0.8 & 49 & 5.88 & 54.88 & 65 \\
\hline 9 & Pocket Tack & I & SNLS & & 0.35 & 21 & 2.52 & 23.52 & 153 \\
\hline 10 & Shoulder Join (2) & 1 & OL & & 0.3 & 18 & 2.16 & 20.16 & 178 \\
\hline 11 & Sleeve Cuff Servicing \& join & I & OL & & 0.4 & 27 & 3.24 & 30.24 & 119 \\
\hline 12 & Sleeve Cuff Top Stitch & 1 & $\mathrm{FL}$ & & 0.3 & 18 & 2.16 & 20.16 & 178 \\
\hline 13 & Sleeve join & 1 & $\mathrm{OL}$ & & 0.3 & 18 & 2.16 & 20.16 & 178 \\
\hline 14 & Arm Hole TS (2) & 1 & $\mathrm{FL}$ & & 0.3 & 18 & 2.16 & 20.16 & 178 \\
\hline 15 & Side seam join (2) & 1 & OL & & 0.55 & 35 & 4.2 & 39.2 & 91 \\
\hline 16 & $\begin{array}{l}\text { Pannel join at bottom rib } \\
\text { (2) }\end{array}$ & 1 & $\mathrm{OL}$ & & 0.3 & 18 & 2.16 & 20.16 & 178 \\
\hline 17 & Pannel mouth TK (2) & 1 & SNLS & & 0.22 & 11 & 1.32 & 12.32 & 292 \\
\hline 18 & Pannel TS (2) & 1 & SNLS & & 0.3 & 18 & 2.16 & 20.16 & 178 \\
\hline 19 & $\begin{array}{l}\text { Bottom rib join position } \\
\text { mark \& Rib join }\end{array}$ & 1 & $\mathrm{O} / \mathrm{L}$ & & 0.76 & 47 & 5.64 & 52.64 & 68 \\
\hline 20 & Bottom rib top stitch & 1 & $\mathrm{FL}$ & & 0.6 & 35 & 4.2 & 39.2 & 91 \\
\hline 21 & $\begin{array}{l}\text { Zipper cover mark \& cover } \\
\text { make }\end{array}$ & 1 & SNLS & & 0.27 & 18 & 2.16 & 20.16 & 178 \\
\hline 22 & Zipper cover turn \& TS & 1 & SNLS & & 0.3 & 18 & 2.16 & 20.16 & 178 \\
\hline 23 & Zipper COVER ATT & 1 & SNLS & & 0.22 & 11 & 1.32 & 12.32 & 292 \\
\hline 24 & Zipper piping (2) operation & I & $\mathrm{FL}$ & & 0.3 & 18 & 2.16 & 20.16 & 178 \\
\hline 25 & Zipper edge fold \& TK (2) & I & $\mathrm{FL}$ & & 0.2 & 11 & 1.32 & 12.32 & 292 \\
\hline 26 & Zipper ATT - Left & 1 & SNLS & & 0.4 & 27 & 3.24 & 30.24 & 119 \\
\hline 27 & Zipper ATT - Right & 1 & SNLS & & 0.4 & 27 & 3.24 & 30.24 & 119 \\
\hline 28 & Collar inner part rolling & 1 & SNLS & & 0.25 & 15 & 3 & 18 & 200 \\
\hline 29 & $\begin{array}{l}\text { Collar inner part mark \& } \\
\text { join }\end{array}$ & & SNLS & & 0.25 & 13 & 1.56 & 14.56 & 247 \\
\hline 30 & Collar mark \& join & 1 & SNLS & & 0.22 & 12 & 1.44 & 13.44 & 267 \\
\hline 31 & Back tape piping & 1 & $\mathrm{~F} / \mathrm{L}$ & & 0.22 & 12 & 1.44 & 13.44 & 267 \\
\hline 32 & $\begin{array}{l}\text { BK Tape top stitch\& corner } \\
\text { fold }\end{array}$ & 1 & SNLS & & 0.5 & 30 & 3.6 & 33.6 & 107 \\
\hline \multirow[t]{2}{*}{33} & Final thread trimming & 3 & MNL & 0.5 & & 30 & 3.6 & 33.6 & 107 \\
\hline & Total & 35 & & & 12.77 & & & 896.44 & \\
\hline
\end{tabular}

Table 2 Traditional operation breakdown of knit jacket

\begin{tabular}{|c|c|c|c|c|c|c|c|c|c|}
\hline \multirow[b]{2}{*}{ SL No. } & \multirow[b]{2}{*}{ Operation } & \multirow[b]{2}{*}{ No. of worker } & \multirow[b]{2}{*}{$M / C$} & \multicolumn{2}{|c|}{ STD. SMV } & \multirow{2}{*}{$\begin{array}{l}\text { Actual Time } \\
\text { Sec(AVG.) }\end{array}$} & \multirow[b]{2}{*}{$\begin{array}{l}\text { Allowance } \\
12 \%\end{array}$} & \multirow[b]{2}{*}{$\begin{array}{l}\text { STD.Time } \\
\text { Sec }\end{array}$} & \multirow[b]{2}{*}{ Capacity } \\
\hline & & & & Manual & Manual & & & & \\
\hline I & Pocket bone mark & 1 & MNL & 0.25 & & 17 & 1.44 & 18.44 & 195 \\
\hline 2 & Bone corner & 1 & MNL & 0.42 & & 27 & 3.24 & 30.24 & 119 \\
\hline 3 & Bone attach for pocket & 1 & SNLS & & 0.5 & 32 & 3.84 & 35.84 & 100 \\
\hline 4 & Body mark for pocket & I & MNL & 0.3 & & 21 & 2.52 & 23.52 & 153 \\
\hline 5 & Pocket attach & I & SNLS & & 0.58 & 36 & 4.32 & 40.32 & 89 \\
\hline
\end{tabular}


Table Continues.

\begin{tabular}{|c|c|c|c|c|c|c|c|c|c|}
\hline \multirow[b]{2}{*}{ SL No. } & \multirow[b]{2}{*}{ Operation } & \multirow[b]{2}{*}{ No. of worker } & \multirow[b]{2}{*}{$M / C$} & \multicolumn{2}{|c|}{ STD. SMV } & \multirow{2}{*}{$\begin{array}{l}\text { Actual Time } \\
\text { Sec(AVG.) }\end{array}$} & \multirow{2}{*}{$\begin{array}{l}\text { Allowance } \\
12 \%\end{array}$} & \multirow{2}{*}{$\begin{array}{l}\text { STD.Time } \\
\text { Sec }\end{array}$} & \multirow[b]{2}{*}{ Capacity } \\
\hline & & & & Manual & Manual & & & & \\
\hline 6 & Pocket cut & I & MNL & 0.67 & & 44 & 5.28 & 49.28 & 73 \\
\hline 7 & Pocket top stitch & I & SNL & & 0.4 & 27 & 3.24 & 30.24 & 119 \\
\hline 8 & $\begin{array}{l}\text { Bone inside tack \& } \\
\text { pocket top stitch } \\
\text { lower }\end{array}$ & I & SNLS & & 0.8 & 51 & 6.12 & 57.12 & 63 \\
\hline 9 & Pocket bag close both & I & $\mathrm{OL}$ & & 0.4 & 27 & 3.24 & 30.24 & 119 \\
\hline 10 & Pocket bag mouth & I & SNLS & & 0.8 & 49 & 5.88 & 54.88 & 65 \\
\hline 11 & Pocket tack & I & SNLS & & 0.35 & 21 & 2.52 & 23.52 & 153 \\
\hline 12 & Care label join & I & SNLS & & 0.15 & 6 & 0.72 & 6.72 & 535 \\
\hline 13 & Shoulder join (2) & I & OL & & 0.3 & 18 & 2.16 & 20.16 & 178 \\
\hline 14 & Sleeve cuff servicing & I & OL & & 0.33 & 21 & 2.52 & 23.52 & 153 \\
\hline 15 & Sleeve cuff join (2) & I & OL & & 0.33 & 20 & 2.4 & 22.4 & 160 \\
\hline 16 & Sleeve cuff top stitch & I & $\mathrm{FL}$ & & 0.3 & 18 & 2.16 & 20.16 & 178 \\
\hline 17 & Arm hole TS (2) & I & $\mathrm{FL}$ & & 0.3 & 18 & 2.16 & 20.16 & 178 \\
\hline 18 & Side seam join (2) & I & OL & & 0.55 & 35 & 4.2 & 39.2 & 91 \\
\hline 19 & $\begin{array}{l}\text { Pannel join at bottom } \\
\text { RIB (2) }\end{array}$ & I & OL & & 0.3 & 18 & 2.16 & 20.16 & 178 \\
\hline 20 & Pannel mouth TK (2) & I & SNLS & & 0.22 & 11 & 1.32 & 12.32 & 292 \\
\hline 21 & Pannel TS (2) & I & SNLS & & 0.3 & 18 & 2.16 & 20.16 & 178 \\
\hline 22 & $\begin{array}{l}\text { Bottom RIB join } \\
\text { position }\end{array}$ & I & MNL & 0.3 & & 18 & 2.16 & 20.16 & 178 \\
\hline 23 & Bottom RIB join & I & OL & & 0.75 & 45 & 5.4 & 50.4 & 71 \\
\hline 24 & Bottom RIBTS & I & $\mathrm{FL}$ & & 0.6 & 35 & 4.2 & 39.2 & 73 \\
\hline 25 & Zipper cover mark & I & MNL & 0.12 & & 6 & 0.72 & 6.72 & 535 \\
\hline 26 & Zipper cover make & I & SNLS & & 0.25 & 17 & 2.04 & 19.04 & 189 \\
\hline 27 & Zipper cover turn \& & I & SNLS & & 0.3 & 18 & 2.16 & 20.16 & 178 \\
\hline 28 & Zipper cover ATT & I & SNLS & & 0.22 & 12 & 1.44 & I3.44 & 267 \\
\hline 29 & $\begin{array}{l}\text { Zipper piping (2) } \\
\text { operation }\end{array}$ & I & $\mathrm{FL}$ & & 0.3 & 20 & 2.4 & 22.4 & 160 \\
\hline 30 & $\begin{array}{l}\text { Zipper edge fold \& } \\
\text { TK (2) }\end{array}$ & I & $\mathrm{FL}$ & & 0.2 & 11 & 1.32 & 12.32 & 292 \\
\hline 31 & Zipper ATT- left & I & SNLS & & 0.4 & 24 & 2.88 & 26.88 & 133 \\
\hline 32 & Zipper ATT- right & I & SNLS & & 0.4 & 24 & 2.88 & 26.88 & 133 \\
\hline 33 & Collar inner part & I & SNLS & & 0.25 & 13 & 1.56 & 14.56 & 247 \\
\hline 34 & Collar inner part mark & I & MNL & 0.25 & & 13 & 1.56 & 14.56 & 247 \\
\hline 35 & Collar 2 part join & I & OL & & 0.22 & 14 & 1.68 & 15.68 & 229 \\
\hline 36 & Collar mark for join & I & MNL & 0.22 & & 14 & 1.68 & 15.68 & 229 \\
\hline 37 & Collar join & I & SNLS & & 0.5 & 30 & 3.6 & 33.6 & 107 \\
\hline 38 & BK tape Piping & I & $\mathrm{FL}$ & & 0.4 & 20 & 2.4 & 22.4 & 160 \\
\hline 39 & $\begin{array}{l}\text { BK tape TSW/corner } \\
\text { fold }\end{array}$ & I & SNLS & & 0.5 & 30 & 3.6 & 33.6 & 107 \\
\hline \multirow[t]{2}{*}{40} & Final thread trimming & 3 & MNL & 0.5 & & 30 & 3.6 & 33.6 & 107 \\
\hline & Total & 42 & & & 15.43 & & & 1013.88 & \\
\hline
\end{tabular}




\section{Transportation Analysis (Table 3) (Figure 3)}

Table 3 Transportation analysis

\begin{tabular}{|c|c|c|c|c|c|c|}
\hline KPI & Unit of measure & Traditional line & Avg. & Lean line & Avg. & Improvement \\
\hline \multirow{5}{*}{ Transportation } & \multirow{5}{*}{ Feet } & 351 & \multirow{5}{*}{345} & 145 & \multirow{5}{*}{143} & \multirow{5}{*}{$58.55 \%$} \\
\hline & & 350 & & 143 & & \\
\hline & & 348 & & 144 & & \\
\hline & & 350 & & 143 & & \\
\hline & & 349 & & 142 & & \\
\hline
\end{tabular}

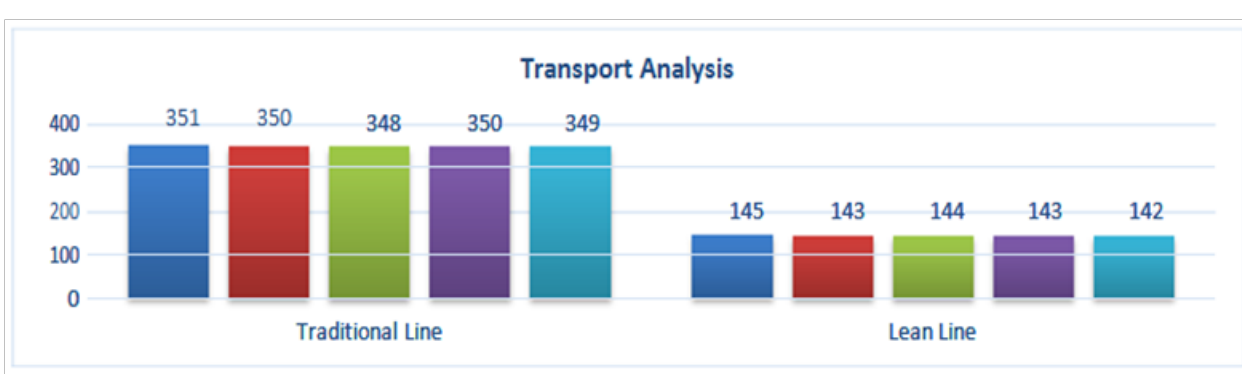

Figure 3 Transport analysis traditional vs lean line.

\section{WIP Analysis (Table 4) (Figure 4)}

Table 4 WIP analysis

\begin{tabular}{|c|c|c|c|c|c|c|}
\hline KPI & Unit of measure & Traditional Line & Avg. & Lean Line & Avg. & Improvement \\
\hline \multirow{5}{*}{ Inventory/WIP } & \multirow{5}{*}{ Quantity } & 815 & \multirow{5}{*}{813} & 400 & \multirow{5}{*}{400} & \multirow{5}{*}{$50.79 \%$} \\
\hline & & 810 & & 398 & & \\
\hline & & 812 & & 402 & & \\
\hline & & 816 & & 396 & & \\
\hline & & 810 & & 402 & & \\
\hline
\end{tabular}

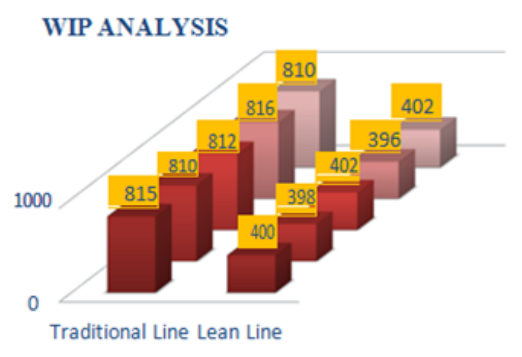

Figure 4 WIP analysis traditional vs lean line.

\section{Space utilization analysis (Table 5) (Figure 5)}

Table 5 Space utilization analysis

\begin{tabular}{lllllll}
\hline KPI Space & Unit of measure & Traditional line & Avg. & Lean line & Avg. & Improvement \\
\hline & & 5.77 & & 4.62 & & \\
Utilization & Minute & 5.6 & 4.5 & & \\
& & 4.96 & 5.55 & 4.45 & 4.52 & $18.55 \%$ \\
& 5.1 & & 4.62 & & \\
\hline
\end{tabular}




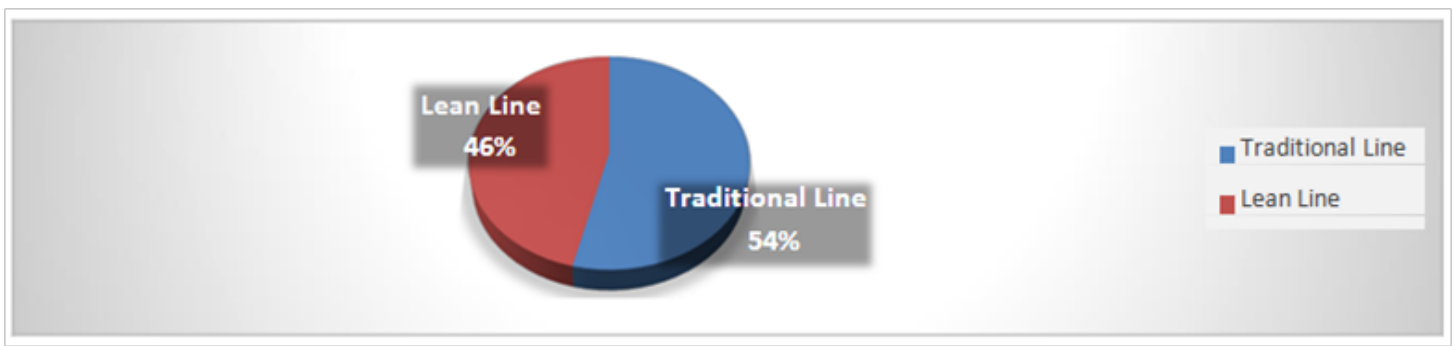

Figure 5 Space utilization traditional vs lean line.

\section{Workstation analysis (Table 6) (Figure 6)}

Table 6 Workstation analysis

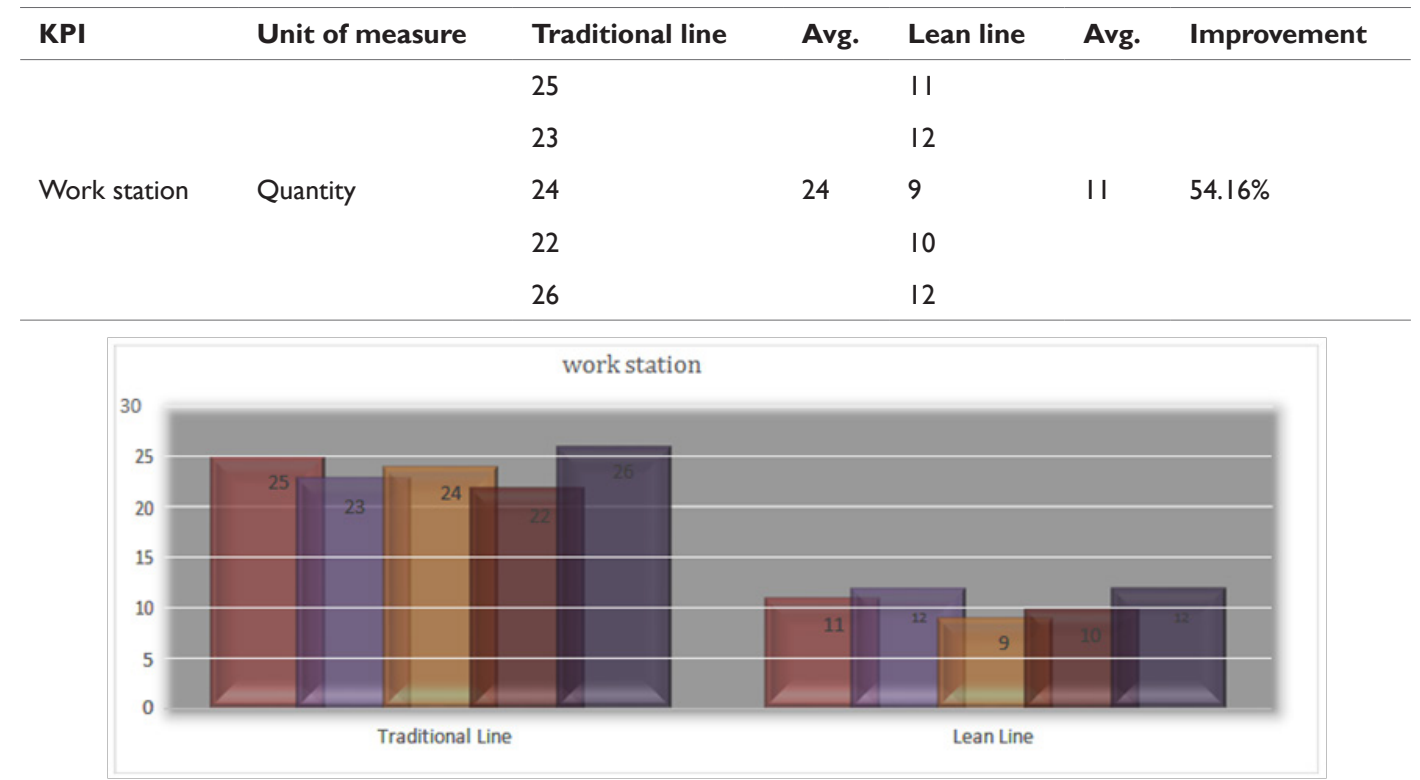

Figure 6 Work station traditional vs lean line.

\section{Defects Analysis (Table 7) (Figure 7)}

Table 7 Defects analysis

\begin{tabular}{lll}
\hline Defects & Traditional line & Lean line \\
\hline Seam Puckering & 30 & 18 \\
Slipped stitch & 27 & 9 \\
Staggered stitch & 18 & 5 \\
Thread Breakage & 16 & 8 \\
Variable Stitch density & 27 & 11
\end{tabular}

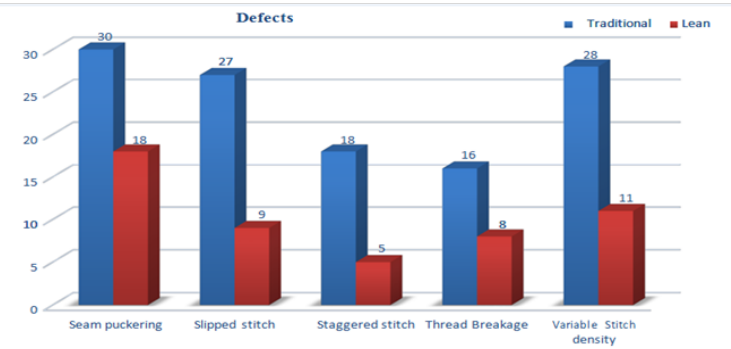

Figure 7 Defects in traditional vs lean line.

\section{Comparing key productivity metrics}

We have used time study to balance these sewing lines which is a part of work study. It implements the use of SMV calculation to identify the points where production has gone below the standard level and the places where the production is above the standard. Then it is balanced to remove bottle necks in order to increase productivity. Considerable improvement observed by using time study as a line balancing technique changing from traditional layout to balance layout model (Table 8) (Table 9).

Table 8 Comparing key productivity indicator

\begin{tabular}{lllll}
\hline Topics & $\begin{array}{l}\text { Unit of } \\
\text { measure }\end{array}$ & $\begin{array}{l}\text { Traditional } \\
\text { line }\end{array}$ & $\begin{array}{l}\text { Lean } \\
\text { line }\end{array}$ & Improvement \\
\hline $\begin{array}{l}\text { Inventory } \\
\begin{array}{l}\text { Transport } \\
\text { Analysis }\end{array}\end{array}$ & Quantity & 813 & 400 & $50.79 \%$ \\
$\begin{array}{l}\text { Space } \\
\text { utilization }\end{array}$ & Min & 345 & 143 & $58.55 \%$ \\
$\begin{array}{l}\text { Work station } \\
\text { Quantity }\end{array}$ & 24 & 4.55 & $18.55 \%$ \\
\hline
\end{tabular}


Table 9 Productivity analysis

\begin{tabular}{lll}
\hline Topic & Traditional line & Lean line \\
\hline Productivity & $64 \%$ & $78 \%$ \\
Line efficiency & $42.89 \%$ & $55.49 \%$ \\
SMV increased & $9.46 \%$ & $16.99 \%$ \\
SMV target Fulfillment & $64 \%$ & $78 \%$ \\
No of worker & 42 & 35 \\
Bottlenecks & 2 & Nil \\
Capacity/hr utilization & $149 \mathrm{pcs}$ & $140 \mathrm{pcs}$ \\
\hline
\end{tabular}

\section{Conclusion}

For a jacket, using traditional system our input was $100 \mathrm{pcs} / \mathrm{hr}$ and output was $64 \mathrm{pcs} / \mathrm{hr}$ with a productivity of $64 \%$. But when we applied lean system then our input was same but the system was so efficient that we got an increase output of $78 \mathrm{pcs} / \mathrm{hr}$. This is a clear indication for increasing productivity. Lack of knowledge, specifically in production systems and resources management of the operations manager of Garments, resulted to the low productivity and efficiency of manpower. The lean manufacturing system is a continuous improvement method; thereby, its implementation helps the company minimize waste, enhance quality of products and definitely create its sustainability. Lean manufacturing tools contribute to the productivity of both workers and the company. The Time Study monitoring system, an output of the study, is an effective and efficient tool to enhance productivity in the entire sewing section, whose benefits extend to the whole organization.

\section{Findings}

Though the lean technique is new for most of the apparel industry in Bangladesh but if a industry implement this technique it helps them to increase their overall productivity. Key findings are:
a. Best utilization of man, machine, materials
b. Increasing productivity
c. Reduce lead time
d. Reduce wastes
e. Ensure just in time shipment

\section{Recommendations}

i. Though the lean floor consists of cutting, sewing and finishing section, there should be minimum waiting time in fabric cutting section before bulk production starting. It is important to establish traffic light system to reduce the unnecessary transportation.

ii. There should be re-layout of lean floor to reduce the transportation time and also reduce the excess inventory in the line.

iii. Unnecessary movement of man, machine and materials should be avoided to reduce the unwanted motion.

iv. Pattern should be cut as per sewing floor requirements otherwise there may be over production.

v. Reduce the number of process in a line to eliminate the possibility of over-processing as well as eliminate cost due to over-processing.

\section{Acknowledgments}

None.

\section{Funding}

None.

\section{Conflicts of interest}

The authors declare that they have no competing interests.

\section{References}

1. Monden Y. Toyota production system: an integrated approach to just-intime. Institute of Industrial Engineers: CRC Press; 2012.

2. Peter H, Anders B, Jensen PL, et al. Lean and the working environment: a review of the literature. International Journal of Operations \& Production Management. 2012;32(7):829-849.

3. Rachna S, Peter T Ward. Defining and developing measures of lean production. Journal of Operations Management. 2007;25(4):785-805.

4. Silva SKPN. Applicability of value stream mapping (VSM) in the apparel industry in Sri Lanka. International Journal of Lean Thinking. 2012;3(1).

5. David McBride. The 7 manufacturing wastes. EMS Consulting group Inc; 2003.

6. Naresh Paneru. Implementation of lean manufacturing tools in garment manufacturing process focusing sewing section of men's shirt. Master's thesis, Autumn 2011 Degree Programme in Industrial Management Oulu University of Applied Sciences; 2011.

7. Md. Mazedul I, Adnan MK, Md. Monirul I. Application of lean manufacturing to higher productivity in the apparel industry in Bangladesh. International Journal of Scientific \& Engineering Research. 2013;4(2).

8. Afsana H, Talha J, Kamruzzaman K, et al. Prospect of implementation of lean manufacturing for apparel industries in Bangladesh. American Journal of Engineering Research (AJER). 2017;6(9):86-96. 